\title{
A structural equation modelling of the buffering effect of social support on the report of common mental disorders in Zimbabwean women in the postnatal period
}

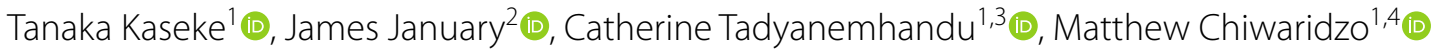
and Jermaine M. Dambi ${ }^{1,4^{*}}$ (1)

\begin{abstract}
Objective: Globally, 13-20\% of women experience a common mental disorder (CMD) postnatally. Unfortunately, the burden of CMDs is disproportionally substantial in women from low-income countries. Nevertheless, there is a growing recognition of the buffering effect of social support (SS) on psychiatric morbidity and the need for mental well-being support services/interventions. This study evaluated the relationship between psychiatric morbidity and SS levels, and factors influencing the mental health functioning of Zimbabwean women postnatally. Data were collected from 340 mothers and were analysed through structural equation modelling.

Results: The mothers' mean age was 26.6 (SD 5.6) years. The mean Multidimensional Scale of Perceived Social Support score was 42.7 (SD 10.8), denoting high levels of SS. Additionally, 29.1\% of the population reported excessive psychiatric morbidity, the median Shona Symptoms Questionnaire score was 5 (IQR: 2-8). The structural equation model demonstrated the buffering effects of SS on psychiatric morbidity ( $r=-0.585, p=0.01)$, and accounted for $70 \%$ of the variance. Being unmarried, increased maternal age, lower educational and income levels were associated with poorer maternal mental health. There is a need for routine; surveillance and treatment of CMDs in women in the postnatal period, including integration of low-cost, evidenced-based and task-shifting SS interventions.
\end{abstract}

Keywords: Women, Postnatal, Social support, Mental health, Zimbabwe

\section{Introduction}

Globally, between 13 and $20 \%$ of women who have just given birth experience a mental disorder [1]. Postnatal depression is particularly endemic and is a leading cause of disability in child-bearing women [2]. Other postnatal mental disorders such as anxiety, postnatal blues and psychosis are also prevalent [3]. Unfortunately, the burden of common mental disorders (CMDs) such as maternal depression is disproportionally higher in low-income countries as opposed to high-income countries with

\footnotetext{
*Correspondence: jermainedambi@gmail.com; dmbjer001@myuct.ac.za 1 Department of Rehabilitation, University of Zimbabwe, College of Health Sciences, P.O Box A178, Avondale, Harare, Zimbabwe Full list of author information is available at the end of the article
}

estimated prevalence rates of $19.8 \%$ and $10 \%$ respectively [4]. For example, 30-34.2\% of urban-dwelling, Zimbabwean women suffer from postnatal depression (PND) [5-7]. Poverty, lower education, compromised physical health, a history of a CMD, intimate partner violence, inadequate social support, and changing cultural practices are important predictors to poor mental health status in women who have just given birth [6, 8-11].

Despite the significant burden of CMDs among women in Sub-Saharan Africa, in-depth information on mental health issues in the postnatal period is limited [7, 12]. Nevertheless, there is a growing recognition of the importance of social support (SS) in improving the mental health of women in the postnatal period [11, 13]. For instance, the buffering hypothesis postulates that 
effective psychological and social resources, particularly social stability, social participation, adequate emotional and instrumental support, can be considered protective, i.e. they buffer the impact of life stress on the psychological well-being of the mother $[14,15]$. On the contrary, a lack of SS can lead to adverse outcomes such as; low birth weight, preterm labour, foetal neural tube defects, depression and anxiety [16]. However, there is a paucity of information on the extent to which SS influences maternal mental health in low resource-settings [7]. The current study therefore set out to identify sources of SS and evaluate the buffering effects of SS on the report of CMDs in urban-dwelling, Zimbabwean women in the postnatal period.

\section{Main text}

\section{Study design, research setting and participants}

We conducted a cross-sectional study at Harare City Council primary health centres. The clinics offer a variety of health services including: curative, maternity and postnatal care. Six clinics were purposively selected to ensure recruitment of participants across the socio-economic continuum. Two of the six clinics were in low to medium density catchment areas with four clinics being located in high-density suburbs [17]. Assuming a 33\% prevalence of PND in urban-dwelling, Zimbabwean women [6], the minimal sample size was 340 at $95 \%$ confidence interval and $80 \%$ goal power. Women who were seeking postnatal services and willing to participate on the day of data collection were conveniently selected. Included were biological mothers $\geq 18$ years with children aged 52 weeks and below. Mothers with a confirmed diagnosis of a mental health disorder and or suffering from long-term health conditions such as HIV/AIDS, cancer, among others were similarly excluded as this could have confounded the study outcomes. Mothers not proficient in either English or Shona languages were similarly excluded due to lack of financial resources for translating study outcomes into other languages.

\section{Study instruments}

A purpose-built questionnaire was used to capture the participants' age, gender, marital status, educational level, employment status and perceived level(s) of income. The Shona Symptom Questionnaire (SSQ), an indigenous generic screen, was used to evaluate the report of CMDs in the past 7 days. The SSQ is a binary outcome i.e. "yes" and "no" responses are scored as one and zero respectively. The score range is $0-14$ and scores $\geq 8$ indicate risk of CMDs. The SSQ is especially sensitive in screening for depression and anxiety and has been extensively validated in the research setting $[18,19]$. The Multidimensional Scale of Perceived Social Support (MSPSS), a 12-item outcome was used to measure SS. Respondents rate the extent of satisfaction with the SS received from friends, family and significant other. Responses are ranked on a five-point Likert scale which ranges from "strongly disagree $=1$ " to "strongly agree $=5$ ". The MSPSS is one of the extensively used SS outcomes [20] and has been translated and validated into Shona (a Zimbabwean native language) [21, 22].

\section{Procedure}

After receiving ethical and institutional approvals, the principal investigator (TK) approached prospective participants in the treatment waiting area(s). The researcher explained the study rationale, applied the selection criteria in recruiting participants and afterwards issued a detailed information sheet to mothers meeting the inclusion criteria. Mothers were obliged to provide written consent to participate in the study. All outcomes were primarily self-administered, however, the principal investigator aided participants where necessary.

\section{Data analysis and management}

Data were entered into Microsoft Excel and analysed using STATA (Version 15). Normality was checked using the Shapiro-Wilk Test. Descriptive statistics (frequencies and means) were used to describe participants' sociodemographics and responses on the SSQ and MSPSS. Thereafter, univariate analysis (t-tests, co-relation coefficiencies and analysis of variance tests) was applied to determine factors influencing mothers' mental health. Contextual factors (patients characteristics) and study primary outcomes (SSQ and MSPSS sub-scores) were then entered into the structural equation model as endogenous and exogenous variables respectively. The following parameters were set as a minimum criterion for model fit; Likelihood Ratio Chi squared Test $\left(\chi m s^{2}\right)$-criterial value: $p>0.05$, root mean square error of approximation (RMSEA) - criterial value: $\leq 0.06$, Comparative Fit Index (CFI) - criterial value: $\geq 0.90$, Tucker-Lewis Index (TLI) - criterial value: $\geq 0.90$ and the standardized root mean square residual (SRMR) - criterial value: $\leq 0.06[23]$.

\section{Results}

Many of the mothers were; married (56.8\%), attained secondary education (83.4\%), unemployed (65\%) and reported of medium levels of income (55.3\%). Their children were mostly males (50.9\%), with an average age of 22.6 (SD 13) weeks. Mothers received the least and greatest amount of social support from friends and family respectively, and the mean MSPSS score was 42.7 (SD 10.8), denoting high levels of SS. Additionally, 29.1\% of the mothers showed excessive psychiatric morbidity and 
the median SSQ score was 5 (IQR: 2-8) (Table 1). See Additional files 1 and 2 for frequencies of reported problems on the MSPSS and SSQ respectively.

Illustrated in Fig. 1 is the model explaining the buffering effects of social support on psychiatric morbidity $(r=-0.585, \mathrm{p}=0.01)$ and the associated contextual factors. The model accounted for $70 \%$ of the variance (See Additional file 3) and displayed excellent fit as outlined in Table 2. Being unmarried, lower education status, lower income level, and increased maternal age were associated with poorer maternal mental health.

\section{Discussion}

Consistent with previous studies, outcomes from the present study suggests that mothers who received a greater amount of SS were likely to have optimal mental health $[11,13]$. Lack of SS is a demonstrated risk factor for psychiatric symptomatology in the postnatal period [24, 25]. Significant others and family were cited as the greatest sources of SS with friends providing the least support. Previous studies have shown that it is not always possible for women to differentiate the effects of spousal support from other kinship members. In collectivist cultures like Zimbabwe, the terms husband/significant other and family are habitually used interchangeably [26]. Further, mothers were likely to have decreased networking opportunities due to the demands of caring for the new infant, and this may further explain the discrepancies in sources of SS [25, 27].

The prevalence of CMDs (29.1\%) was relatively higher compared to the global lifetime prevalence of 18\% [28], and a $16 \%$ prevalence yielded from an almost similar, previous local study [29]. The changing patterns of mental health symptomatology in Zimbabwe especially given the advent of the HIV/AIDS pandemic and the worsening economic challenges the country has been facing may account for the dissimilarity [7]. Poverty, poor nutrition, inmate partner violence, history of depression, lack of spousal support, unstable marital status, unplanned pregnancies and increased social responsibilities are risk factors for increased psychiatric morbidity in the postnatal period for women residing in low-resource settings [11, 13, 24, 30-33].

In our study, having fewer resources (lower education and lower income), small social network (being unmarried) and maternal characteristics (increased maternal age) negatively influenced maternal mental health. Married and cohabiting mothers showed the least risk of psychiatric morbidity. Traumatic experiences such as the death of a loved one, losing a job and relationship

Table 1 Participants descriptive statistics, $\mathbf{N}=\mathbf{3 4 0}$

\begin{tabular}{|c|c|c|}
\hline Variable & Attribute & Frequency, n (\%) \\
\hline Age of child in weeks ${ }^{a}$ & Mean (SD) & 22.6 (SD 13.0) \\
\hline \multirow[t]{2}{*}{ Gender of child } & Female & $167(49.1)$ \\
\hline & Male & $173(50.9)$ \\
\hline Mother's age ${ }^{a}$ & Mean (SD) & $26.6(5.6)$ \\
\hline \multirow[t]{3}{*}{ Marital status } & Married & $193(56.8)$ \\
\hline & Co-habiting & $101(29.7)$ \\
\hline & Other & $46(13.5)$ \\
\hline \multirow[t]{3}{*}{ Level of education } & Primary & $20(5.8)$ \\
\hline & Secondary & $286(83.4)$ \\
\hline & Tertiary & $34(9.9)$ \\
\hline \multirow[t]{3}{*}{ Employment status } & Formally employed & $40(11.8)$ \\
\hline & Self-employed & $77(22.6)$ \\
\hline & Unemployed & $223(65.0)$ \\
\hline \multirow[t]{3}{*}{ Perceived level of income } & Below average & $88(24.1)$ \\
\hline & Average & $188(55.3)$ \\
\hline & Above average & $70(20.6)$ \\
\hline \multirow[t]{4}{*}{ Social support (MSPSS) scores ${ }^{a}$} & Family [mean (SD)] & 3.8 (SD 0.9) \\
\hline & Friends [mean (SD)] & 3.1 (SD 1.2) \\
\hline & Significant other [mean (SD)] & 3.8 (SD 1.0) \\
\hline & Summative score [mean (SD)] & 42.7 (SD 10.8) \\
\hline \multirow[t]{2}{*}{ Psychiatric morbidity (SSQ) scores ${ }^{a}$} & SSQ scores $\geq 8[n(\%)]$ & $99(29.1 \%)$ \\
\hline & Summative score: median $\left[\mathrm{Q}_{1}-\mathrm{Q}_{3}\right]$ & 5 [IQR: 2-8] \\
\hline
\end{tabular}

${ }^{a}$ Results not presented in the $\mathrm{n}(\%)$ format 


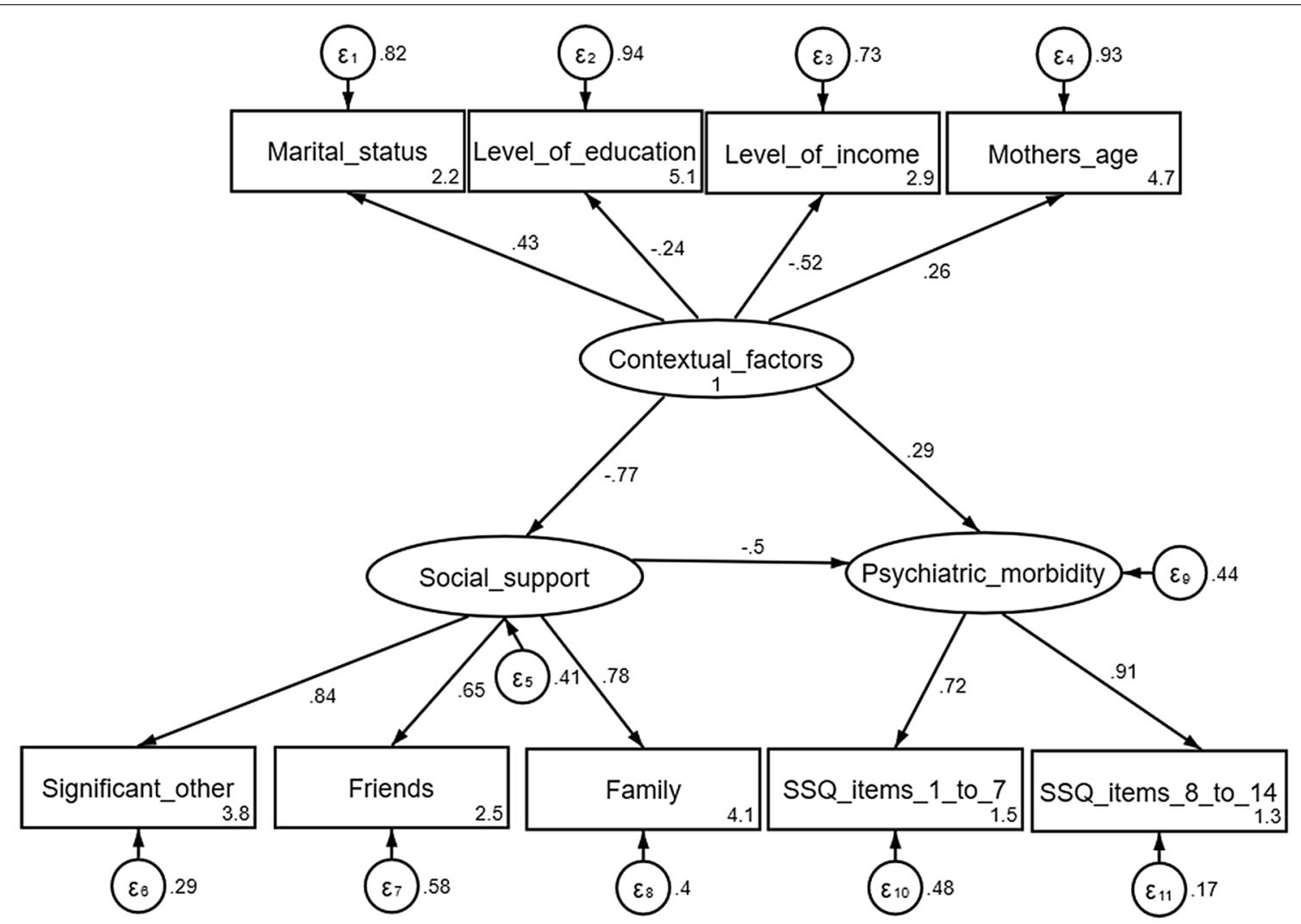

Fig. 1 Mothers' mental health model showing the relationship between perceived levels of social support, report of common mental disorders and contextual/demographic factors

Table 2 Model fit indices, $\mathbf{N}=\mathbf{3 4 0}$

\begin{tabular}{|c|c|c|c|}
\hline Fit statistic & Index & Criterion for fit & Result-interpretation \\
\hline \multirow[t]{2}{*}{ Likelihood ratio } & Chi squared test $\left(x m s^{2}\right)$ & $p>0.05$ & $x^{2}(\mathrm{df} 24)=84.87, p<0.001$-misfit \\
\hline & Normed Chi square $\left[x^{2} / d f\right]$ & $x^{2} / d f<2$ & 3.5-misfit \\
\hline Population error & Root mean squared error of approximation (RMSEA)-(90\% Cl) & RMSEA $\leq 0.06$ & 0.054 (0.026: 0.080)—good fit \\
\hline \multirow[t]{2}{*}{ Information criteria } & Akaike's information criterion (AIC) & The smaller, the better & 8965.5 - best fit \\
\hline & Bayesian information criterion (BIC) & The smaller, the better & 9080.32-best fit \\
\hline \multirow[t]{2}{*}{ Baseline comparison } & Comparative Fit Index (CFI) & $C F I \geq 0.90$ & 0.928 - good fit \\
\hline & Tucker-Lewis Index (LFI) & $L F I \geq 0.90$ & 0.893 -good fit \\
\hline \multirow[t]{2}{*}{ Size of residuals } & Standardized root mean squared residual (SRMR) & $S R M R \leq 0.08$ & 0.056 -good fit \\
\hline & The coefficient of determination (SD) & The greater, the better & 0.7 -good fit \\
\hline
\end{tabular}

breakdown or divorce are associated with poor mental health functioning [34-36]. These events are suggested to reflect additional stress after childbirth, at a time during which women are especially vulnerable [36, 37]. Mothers with higher levels of education reported higher levels of SS. Being educated is an important predictor to greater political and social engagement [38]. Education increases the sense of control that an individual feel over their life and concomitantly increases the chances of accessing stable relationships and expanded social networks which ultimately enhances the amount of the SS received [36, 38]. Further, educated mothers are highly likely to be employed and our findings also revealed that mothers with higher levels of perceived income indicated the least risk of psychiatric morbidity. These findings are in keeping with a previous systematic review which revealed that socio-economic disadvantaged women are five times predisposed to CMDs in the perinatal period [1]. 
Current evidence also suggests that increased maternal age is a risk factor for CMDs and this is in contradiction to previous studies [6, 24, 30, 32, 33]. It has been previously hypothesized that younger mothers are at an increased risk for CMDs as they may not be fully prepared for the parenting role. Further, in certain instances, the lack of SS especially spousal support, may predispose younger mothers to poor mental health functioning as some of the pregnancies maybe unplanned [24, 30]. Older mothers are likely to have greater financial resources, greater education and more likely to be mature and these are protective factors against CMDs according to the buffering hypothesis [39]. On the contrary, fertility problems, delayed parity, and prior obstetric complications are likely to predispose older mothers to CMDs [31, 40]. Further, older mothers may not receive adequate SS in comparison to first-time mothers, older mothers may be deemed "proficient" in infant care, and this predisposes them to an increased risk of CMDs [31, 40]. Other studies did not find any association between maternal age and CMDs $[6,35,39,41]$. Considering the inconclusive evidence from literature, there is a need for further longitudinal and qualitative studies to understand the effects of maternal age on the prevalence of CMDs further.

Collectively, our study outcomes point out the need for the provision of support services such as professional counselling for the improvement of the mental health of mothers in the postnatal period. However, the lack of human resources is a massive threat towards the closure of the huge mental health treatment gap in low-resource settings [42]. This therefore calls for the integration of low-cost, evidenced-based and task-shifting interventions such as the Friendship Bench (FB) [43] in mitigating the burden of CMDs in this populace. The FB concept is centred on the use of trained, lay-persons (grandmothers) in providing standardised, problem-solving therapy (psycho-social support intervention) to persons in need of mental health services. The FB concept is in keeping with the buffering hypothesis which postulates that increased SS is associated with improved mental health $[14,15]$. The FB has been successfully implemented in mitigating the effects of social stigma in individuals suffering from CMDs in the Zimbabwean context [44], and we believe the concept can be successfully integrated into routine postnatal care.

\section{Conclusion}

The prevalence of CMDs was $29.1 \%$ and mothers who received an adequate amount of SS showed optimal mental health. Being unmarried, lower education status, lower income level, and increased maternal age were associated with poorer maternal mental health. There is need for routine surveillance and treatment of CMDs in women in the postnatal period. More importantly, there is also need for integration of low-cost, evidenced-based and task-shifting interventions such as the Friendship Bench [44] in mitigating the burden of CMDs in this populace.

\section{Limitations}

- Causality cannot be inferred as data were collected cross-sectionally.

- Purposively selection of study sites and participants may have introduced selection bias.

- Clinical data used in applying the selection criterion were self-reported.

- Institution-based participant recruitment may have precluded selection of community-dwelling mothers at risk of poor mental health.

\section{Additional files}

Additional file 1. Frequencies of responses on the MSPSS, $N=340$. Table denotes frequencies of responses on the MSPSS, a 12-item social support outcome measure. Responses are rated on a five-point Likert scale, ranging from "strongly disagree $=1$ " to "strongly agree $=5$ ".

Additional file 2. Frequencies of responses on the SSQ, N=340. Table denotes frequencies of responses on the SSQ, a 14-item, binary common mental disorders (CMDs) screen. Respondents indicate if they had experienced any of the enlisted symptoms in the last seven days. A yes response is scored as "one" and no as "zero", a score $\geq 8$ is indicative of risk of CMD.

Additional file 3. Variance explained by the model. Table denotes the variance accounted by the variables and the total model expressing the relationship between contextual factors, levels of perceived social support and report of common mental disorders.

\section{Abbreviations}

AIDS: acquired immune deficiency syndrome; CFI: Comparative Fit Index; CMDs: common mental disorders; HIV: human immunodeficiency virus; JREC: Joint Research and Ethics Committee for the University of Zimbabwe, College of Health Sciences \& Parirenyatwa Group of Hospitals; MSPSS: Multidimensional Scale of Perceived Social Support; PND: postnatal depression; RMSEA: root mean square error of approximation; SD: standard deviation; SRMR: standardized root mean square residual; SS: social support; SSQ: Shona Symptom Questionnaire; TLI:Tucker-Lewis Index.

\section{Authors' contributions}

TK, MC and JMD developed the concept and design of the study. TK collected the data and drafted the first version of the manuscript. JMD conducted the data analysis and statistical interpretation, revised the first version of the manuscript, prepared all prerequisite processes for articles submission, submitted the manuscript and is the corresponding author. JJ, CT and MC critically appraised/peer-reviewed and made substantive contributions on the second to fifth versions of the manuscript in preparation for submission to the journal. All authors read and approved the final manuscript. 


\begin{abstract}
Author details
${ }_{1}^{1}$ Department of Rehabilitation, University of Zimbabwe, College of Health Sciences, P.O Box A178, Avondale, Harare, Zimbabwe. ${ }^{2}$ Department of Community Medicine, University of Zimbabwe, College of Health Sciences, P.O Box A178, Avondale, Harare, Zimbabwe. ${ }^{3}$ Department of Physiotherapy, School of Therapeutic Sciences, Faculty of Health Sciences, University of the Witwatersrand, Johannesburg, South Africa. ${ }^{4}$ School of Health and Rehabilitation Sciences, Faculty of Health Sciences, University of Cape Town Observatory, Cape Town 7700, South Africa.
\end{abstract}

\section{Acknowledgements}

We would want to acknowledge participants for their invaluable participation especially. The data were collected as part of TK's undergraduate thesis which JMD supervised her. Appreciation also goes to the AMARI consortium for various capacity building initiatives which facilitated the writing of the present manuscript. The manuscript is a product of the manuscript writing and systematic review workshops facilitated by Dr. Helen Jack (Harvard University/Kings College London). Further, the manuscript is also a practical application of the Academic Career Enhancement Series (ACES) program led by Dr. Christopher Merritt (Kings College London). The senior author utilized the skills acquired through the ACES program in both thesis supervision and mentoring of the first author in producing the first draft of the manuscript. Statistical skills learnt from the data analysis workshops by Dr. Lorna Gibson and Professor Helen Weiss (London School of Hygiene and Tropical Medicine) were also fundamental in enhancing the senior authors'statistical analysis and interpretation skills.

\section{Competing interests}

The authors declare that they have no competing interests.

\section{Availability of data and materials}

The datasets used and/or analysed during the current study are available from the corresponding author on reasonable request. The datasets will be availed onto online repositories once all manuscripts related to the study have been published online.

\section{Consent for publication}

Not applicable as the manuscript does not contain any data from any individual person.

\section{Ethics approval and consent to participate}

Ethical approval for the study was granted by the City of Harare Health Department and the Joint Research and Ethics Committee for the University of Zimbabwe, College of Health Sciences \& Parirenyatwa Group of Hospitals (Ref: JREC/362/17). Participants were treated as autonomous agents and were requested to sign written consent before participation. Pseudo-names were used to preserve confidentiality, data were stored securely, and only the researchers had access to the information gathered, and participants could voluntarily withdraw from the study at any time without any consequences.

\section{Funding}

The MSPSS was adapted, translated and validated into Shona as part of the senior authors' Ph.D. work at the University of Cape Town. The work is being funded by The African Mental Health Research Initiative (AMARI). AMARI is a consortium of four African universities whose overall goal is to build excellence in leadership, training and science amongst African scholars in mental, neurological and substance use (MNS) research in Ethiopia, Malawi, South Africa and Zimbabwe. AMARI, at the University of Zimbabwe College of Health Sciences (UZCHS), secured funding from the Wellcome Trust through the Developing Excellence in Leadership and Science (DELTAS) Africa initiative. The DELTAS Africa Initiative is an independent funding scheme of the African Academy of Sciences (AAS)'s Alliance for Accelerating Excellence in Science in Africa (AESA) and supported by the New Partnership for Africa's Development Planning and Coordinating Agency (NEPAD Agency) with funding from the Wellcome Trust [DEL-15-01] and the UK government. The views expressed in this publication are those of the author(s) and not necessarily those of AAS, NEPAD Agency, Wellcome Trust, or the UK government. The funding agency had no role in the design of the study and collection, analysis, interpretation of data, or in writing the manuscript.

\section{Publisher's Note}

Springer Nature remains neutral with regard to jurisdictional claims in published maps and institutional affiliations.

Received: 7 November 2018 Accepted: 22 February 2019

Published online: 28 February 2019

\section{References}

1. Fisher J, de Mello MC, Patel V, Rahman A, Tran T, Holton S, et al. Prevalence and determinants of common perinatal mental disorders in women in low-and lower-middle-income countries: a systematic review. Bull World Health Organ. 2012;90:139-49.

2. Keynejad RC, Dua T, Barbui C, Thornicroft G. WHO Mental Health Gap Action Programme (mhGAP) Intervention Guide: a systematic review of evidence from low and middle-income countries. Evid Based Ment Health. 2018;21:30-4.

3. Sockol LE, Epperson CN, Barber JP. The relationship between maternal attitudes and symptoms of depression and anxiety among pregnant and postnatal first-time mothers. Arch Womens Ment Health. 2014;17:199-212

4. Gelaye B, Rondon M, Araya R, Williams MA. Epidemiology of maternal depression, risk factors, and child outcomes in low-income and middleincome countries. Lancet Psychiatry. 2016;3:973-82.

5. January J, Mutamba N, Maradzika J. Correlates of postnatal depression among women in Zimbabwean semi-urban and rural settings. J Psychol Afr. 2017;27:93-6.

6. January J, Chivanhu H, Chiwara J, Denga T, Dera K, Dube T, et al. Prevalence and corelates of postnatal depression in an urban high density surburb of Harare. Cent Afr J Med. 2015;61:1-4.

7. Chibanda D, Mangezi W, Tshimanga M, Woelk G, Rusakaniko P, StranixChibanda L, et al. Validation of the Edinburgh postnatal depression scale among women in a high HIV prevalence area in urban Zimbabwe. Arch Womens Ment Health. 2010;13:201-6.

8. Negron R, Martin A, Almog M, Balbierz A, Howell EA. Social support during the postnatal period: mothers' views on needs, expectations, and mobilization of support. Matern Child Health J. 2013;17:616-23.

9. Campbell-grossman C, Brage D, Kevin H, Brown SE, Hanna KM, Yates BC. Low-income, African American, adolescent mothers' depressive symptoms, perceived stress, and social support. J Child Fam Stud. 2016;25:2306-14

10. Lancaster CA, Gold KJ, Flynn HA, Yoo H, Marcus SM, Davis MM. Reviews risk factors for depressive symptoms during pregnancy: a systematic review. Am J Obstet Gynecol. 2010;202:5-14. https://doi.org/10.1016/j. ajog.2009.09.007.

11. Stewart RC, Umar E, Tomenson B, Creed F. Validation of the multi-dimensional scale of perceived social support (MSPSS) and the relationship between social support, intimate partner violence and antenatal depression in Malawi. BMC Psychiatry. 2014;14:180.

12. Chibanda D, Mangezi W, Tshimanga M, Woelk G, Rusakaniko S, StranixChibanda L, et al. Postnatal depression by HIV status among women in Zimbabwe. J Womens Health. 2010;19:2071-7.

13. Stewart RC, Umar E, Tomenson B, Creed F. A cross-sectional study of antenatal depression and associated factors in Malawi. Arch Womens Ment Health. 2014;17:145-54.

14. Glazier RH, Elgar FJ, Goel V, Holzapfel S. Stress, social support, and emotional distress in a community sample of pregnant women. J Psychosom Obstet Gynaecol. 2004;25:247-55.

15. Hayakawa N, Koide T, Okada T, Murase S, Aleksic B. The postnatal depressive state in relation to perceived rearing : a prospective cohort study. PLOS ONE. 2012;7:1-6.

16. Da Costa D, Larouche J, Dritsa M, Brender W. Psychosocial correlates of prepartum and postnatal depressed mood. J Affect Disord. 2000;59:31-40

17. Zimbabwe National Statistic Agency. Zimbabwe population census 2012. www.zimstat.co.zW/.../Census/CensusResults2012/National_Report.pdf. Accessed 25 Aug 2018.

18. Haney E, Singh K, Nyamukapa C, Gregson S, Robertson L, Sherr L, et al. One size does not fi $t$ all: psychometric properties of the Shona Symptom 
Questionnaire (SSQ) among adolescents and young adults in Zimbabwe. J Affect Disord. 2014;167:358-67.

19. Chibanda D, Verhey R, Gibson LJ, Munetsi E, Machando D, Rusakaniko S, et al. Validation of screening tools for depression and anxiety disorders in a primary care population with high HIV prevalence in Zimbabwe. J Affect Disord. 2016;198:50-5.

20. Dambi J, Corten L, Chiwaridzo M, Jack H, Jelsma J, Mlambo T. A systematic review of the psychometric properties of the cross-cultural translations and adaptations of the multidimensional perceived social support scale (MSPSS). Health Qual Life Outcomes. 2018;16:1-19.

21. Dambi JM, Tapera L, Chiwaridzo M, Tadyanemhandu C, Nhunzvi C. Psychometric evaluation of the Shona version of the multidimensional scale of perceived social support scale (MSPSS—Shona) in adult informal caregivers of patients with cancer in Harare, Zimbabwe. Malawi Med J. 2017;29:89-96.

22. Nyoni AM, Chiwaridzo M, Tadyanemhandu C, January J, Dambi JM. Profiling the mental health of diabetic patients : a cross-sectional survey of Zimbabwean patients. BMC Res Notes. 2018;11:1-7.

23. Goodboy AK, Kline RB. Statistical and practical concerns with published communication research featuring structural equation modeling. Commun Res Rep. 2017;34:68-77.

24. Liu S, Yan Y, Gao X, Xiang S, Sha T, Zeng G, et al. Risk factors for postnatal depression among Chinese women: path model analysis. BMC Pregnancy Childbirth. 2017:17:1-7.

25. Miller ML, Kroska EB, Grekin R. Immediate postnatal mood assessment and postnatal depressive symptoms. J Affect Disord. 2017;207:69-75.

26. Lund L, Ross L, Petersen MA, Groenvold M. Cancer caregiving tasks and consequences and their associations with caregiver status and the caregiver's relationship to the patient : a survey. BMC Cancer. 2014;14:1-13.

27. Coates R, Ayers $S$, de Visser R. Women's experiences of postnatal distress: a qualitative study. BMC Pregnancy Childbirth. 2014:14:1-14.

28. Steel Z, Marnane C, Iranpour C, Chey T, Jackson JW, Patel V, et al. The global prevalence of common mental disorders: a systematic review and meta-analysis 1980-2013. Int J Epidemiol. 2014;43:476-93.

29. Nhiwatiwa S, Patel V, Acuda W. Predicting postnatal mental disorder with a screening questionnaire: a prospective cohort study from Zimbabwe. J Epidemiol Community Health. 1998;52:262-6.

30. Khalifa DS, Glavin K, Bjertness E, Lien L. Determinants of postnatal depression in Sudanese women at 3 months postnatal: a cross-sectional study. BMJ Open. 2016;6:e009443.

31. McMahon CA, Boivin J, Gibson FL, Hammarberg K, Wynter K, Fisher JRW. Older maternal age and major depressive episodes in the first two years after birth: findings from the parental age and transition to parenthood Australia (PATPA) study. J Affect Disord. 2015;175:454-62.

32. Bottino MN, Nadanovsky P, Moraes CL, Reichenheim ME, Lobato G. Reappraising the relationship between maternal age and postnatal depression according to the evolutionary theory: empirical evidence from a survey in primary health services. J Affect Disord. 2012;142:219-24.

33. Petrosyan D, Armenian HK, Arzoumanian K. Interaction of maternal age and mode of delivery in the development of postnatal depression in Yerevan, Armenia. J Affect Disord. 2011;135:77-81.

34. Małus A, Szyluk J, Galińska-Skok B, Konarzewska B. Incidence of postnatal depression and couple relationship quality. Psychiatr Pol. 2016;50:1135-46

35. Kim Y, Dee V. Sociodemographic and obstetric factors related to symptoms of postnatal depression in hispanic women in rural California. J Obstet Gynecol Neonatal Nurs. 2018;47:23-31.

36. Lewis BA, Billing L, Schuver K, Gjerdingen D, Avery M, Marcus BH. The relationship between employment status and depression symptomatology among women at risk for postnatal depression. Women's Health. 2017:13:3-9.

37. DeJong H, Fox E, Stein A. Rumination and postnatal depression: a systematic review and a cognitive model. Behav Res Ther. 2016;82:38-49.

38. Amoah PA. Social participation, health literacy, and health and well-being: a cross-sectional study in Ghana. SSM Popul Health. 2018;4:263-70.

39. McMahon CA, Boivin J, Gibson FL, Fisher JRW, Hammarberg K, Wynter K, et al. Older first-time mothers and early postnatal depression: a prospective cohort study of women conceiving spontaneously or with assisted reproductive technologies. Fertil Steril. 2011;96:1218-24.

40. Morgan PA, Merrell JA, Rentschler D, Chadderton H. Triple whammy: women's perceptions of midlife mothering. Am J Matern Nurs. 2012;37:156-62.

41. Iwata H, Mori E, Sakajo A, Aoki K, Maehara K, Tamakoshi K. Prevalence of postnatal depressive symptoms during the first 6 months postnatal: association with maternal age and parity. J Affect Disord. 2016;203:227-32.

42. Roberts T, Esponda GM, Krupchanka D, Shidhaye R, Patel V, Rathod S. Factors associated with health service utilisation for common mental disorders: a systematic review. BMC Psychiatry. 2018;18:1-19.

43. Chibanda D, Weiss HA, Verhey R, Simms V, Munjoma R, Rusakaniko S, et al. Effect of a primary care-based psychological intervention on symptoms of common mental disorders in Zimbabwe: a randomized clinical trial. $J$ Am Med Assoc. 2016;316:2618-26.

44. Munetsi E, Simms V, Dzapasi L, Chapoterera G, Goba N, Gumunyu T, et al. Trained lay health workers reduce common mental disorder symptoms of adults with suicidal ideation in Zimbabwe: a cohort study. BMC Public Health. 2018;18:227.
Ready to submit your research? Choose BMC and benefit from:

- fast, convenient online submission

- thorough peer review by experienced researchers in your field

- rapid publication on acceptance

- support for research data, including large and complex data types

- gold Open Access which fosters wider collaboration and increased citations

- maximum visibility for your research: over $100 \mathrm{M}$ website views per year

At BMC, research is always in progress.

Learn more biomedcentral.com/submissions 